\title{
Fragmenting pictures on the Apple Macintosh computer for experimental and clinical applications
}

\author{
JOAN GAY SNODGRASS, BRADFORD SMITH, and KELLY FEENAN \\ New York University, New York, New York \\ and \\ JUNE CORWIN \\ New York University School of Medicine \\ and Psychiatry Service, New York VA Medical Center, New York, New York
}

\begin{abstract}
A set of procedures implemented in Microsoft BASIC is described that creates fragmented versions of pictures scanned into the Apple Macintosh, stores them as resource files, and presents them in a computerized perceptual memory test. A total of 150 pictures were selected from the Snodgrass and Vanderwart (1980) set for fragmentation. The perceptual memory test provides for five forms of 30 pictures each, divided into two sets of 15 that serve alternately as the training or old set and the new set. A training set of 15 pictures is presented for identification during the first (training) phase of the test. The second (test) phase presents the training pictures again, randomly mixed with 15 new pictures for identification. The performance of 100 subjects on the memory test is presented, along with results for each form. Overall, subjects showed improvement on the task with practice (skill learning), indexed by a decrease in thresholds from the training set to the new set. Subjects also showed large savings for the repeated pictures (perceptual learning), indexed by a decrease in thresholds from the new to the old set.
\end{abstract}

This paper describes a set of procedures for fragmenting picture stimuli, storing them as resource files, and presenting them in a computerized test of perceptual learning that runs on an Apple Macintosh Plus. The test follows the Gollin Picture Test (Gollin, 1960) in spirit. In the Gollin test, subjects are shown a series of pictures from which fragments have been deleted, starting with the most fragmented level. Increasingly more complete versions of each picture are shown until all pictures can be identified. Subjects are then retested on the old items to measure perceptual memory. Although the test was initially developed for use with children, it has been used extensively with clinical populations (e.g., Corkin, 1982), following the demonstration by Warrington and Weiskrantz (1968) that amnesic patients show substantial learning and retention as measured by decreased thresholds for repeated pictures after a delay of as long as $\mathbf{3}$ months. This preserved learning ability in amnesics-along with demonstrations in normal subjects that effects of many variables are dissociated in perceptual, compared with episodic, learning tasks-has led several investigators to postulate

This research was supported in part by a Challenge Grant From New York University, in part by a University Research Initiative Program grant from the Air Force Office of Scientific Research, and in part by the Veterans Administration. Requests for reprints should be sent to J. G. Snodgrass, Department of Psychology, New York University, 6 Washington Place, New York, NY 10003. the existence of two or more independent memory systems (e.g., Graf, Squire, \& Mandler, 1984; Jacoby \& Dallas, 1981; Jacoby \& Witherspoon, 1982; Tulving, 1985), although Jacoby (1983) presented an alternative interpretation of such dissociations.

The Gollin set of fragmented pictures consists of 20 pictures of common objects and animals, and the present set consists of 150 pictures of common objects and animals taken from the Snodgrass and Vanderwart (1980) set of standardized pictures. Each picture in the Gollin set has five levels of fragmentation, whereas each picture in the present set has eight levels.

Several properties of the Gollin set were incorporated in the present set. First, as in Gollin's stimuli, fragmentation is cumulative, so that all fragments in a more degraded version are present in a less degraded version. Second, the same fragments are presented when stimuli are repeated as were presented in the original training. This contrasts with fragmentation algorithms that occur on-line, in which randomly selected portions of the picture are deleted each time (e.g., Vokey, Baker, Hayman, \& Jacoby, 1986). This also contrasts with any procedure in which stimulus degradation is accomplished through temporal limitations, such as brief flashes of intact stimuli on a tachistoscope or CRT. In these cases, the observer's perceptual system introduces random fluctuations in determining which portions of the degraded stimulus are seen. Finally, a limitation of the Gollin set is that the most 
fragmented versions are often easily identifiable, leading to ceiling effects. In contrast, in the present set the most fragmented versions are virtually impossible to identify with no previous exposure.

We believe that an important advantage of the present procedure is the ability to control whether the same or different fragments are presented again during memory testing. Comparisons of the two conditions may clarify differences obtained by researchers in the clinical literature with Gollin pictures as opposed to other perceptual tasks.

The remainder of this paper is divided into two parts. In the first, we describe the set of procedures used to produce the fragmented pictures, and in the second, we describe the results of a study using the basic task.

\section{GENERATING THE STIMULI}

The first step was to select candidate pictures from Snodgrass and Vanderwart (1980) that were considered suitable for fragmenting. We selected pictures having moderate complexity and sufficient area so that distinctly different fragmented images could be created. So, for example, the accordion was excluded because of its high complexity and the needle was excluded because of its lack of area.

The next step was to input the pictures into the Macintosh. Because the pictures were to be initially saved as MacPaint files, we reduced the the size of the original drawings to correspond to the size of a MacPaint window (approximately $246 \times 246$ pixels). The pictures were digitized using the Thunderscan digitizer (distributed by Thunderware, 21 Orinda Way, Orinda, CA 94563), an inexpensive peripheral device for the Macintosh that replaces the ribbon cartridge on the Apple ImageWriter and scans an image on a sheet inserted into the printer carriage. The picture is saved as a MacPaint file. Within MacPaint, the picture can be cleaned up with the aid of Fat Bits, and then is centered within a selection square approximately $246 \times 246$ pixels and copied to the scrapbook.

Pictures are copied or cut from the scapbook onto the clipboard, and then are read from the clipboard by an MBASIC program using the OPEN CLIP:PICTURE statement. The pictures are saved in MBASIC sequential files as string variables consisting of encoded graphics instructions (PICT format), one picture to a file. This storage format conserves space compared to the storage requirements for a bit-mapped image. The average storage requirement for 260 pictures from the Snodgrass and Vanderwart set stored in PICT format as MBASIC sequential files is approximately $3 \mathrm{~K}$. However, a disadvantage of the PICT format is that it takes longer to draw on the screen than a bit-mapped image.

Subsequent forms of the pictures are saved as resource files, using libraries available from Clear Lake Research, 5615 Morningside St. \#127, Houston, TX 77005. (These libraries will be incorporated into version 3.0 of Microsoft BASIC.) Resource files have the following advantages over sequential and random access files: (1) they require less storage space than the other types, (2) they permit pictures to be accessed individually by either name or identification number, and (3) they permit replacement of a single picture (Brooks, 1985).

\section{The Fragmentation Process}

In order to produce fragmented pictures that are sufficiently difficult to identify, we found it necessary to delete fairly large blocks of pixels. By a process of trial and error, we chose a $16 \times 16$ pixel block size, although other sizes in that vicinity would also have been acceptable.

In order to delete cumulatively, and to ensure that each successive fragmentation level has fewer fragments than the next lower level, it was necessary to identify which $16 \times 16$ blocks contain information. Because each picture is drawn within a $246 \times 246$ window, there are a total of 256 blocks (blocks in the rightmost column are truncated). The fragmentation program lays out a grid of $16 \times 16$ blocks, determines which blocks contain black pixels, and stores the locations of these critical blocks. Then the program randomly selects increasing proportions of critical blocks to be erased according to an exponential function, to produce eight levels of fragmentation per picture. Level 8 is the complete picture, and Level 1 is the most fragmented picture. The parameter of the exponential function is adjustable to produce more rapid fragmentation at higher values or slower fragmentation at lower values. It is also possible to vary the number of levels in the fragmentation series.

Because the deleted blocks are determined randomly, a very large set of possible fragmentations is possible. In constructing the fragmented stimuli, we ran through several fragmentations until we identified a "good" one. The sequence of random numbers that determined which blocks were to be deleted at each level were saved so that a particular fragmentation series could be reproduced. Our criteria for a good fragmentation were, first, that the general outline of the picture was retained at the most fragmented level, and second, that critical features that would identify a picture (such as the eyes or tail of an animal) were deleted as soon as possible.

Once an acceptable fragmentation series was identified, the random number array was used to create and store eight levels of fragmentation for each picture. The fragmented pictures are stored in resource files in folders on a stimulus disk. The stimulus disk also contains a masterfile for each set, which has the names and identifying numbers (from Snodgrass and Vanderwart) for each of the pictures in a set, as well as the pictures themselves. The memory test program uses the masterfile to determine the identity of the pictures to be used in a particular condition. The stimulus disk also contains resource files with alternative correct names (variant files) for each picture. These are used by the program to determine whether the subject's response matches any of the correct names for a particular picture. The experimenter can update the variant files by adding additional names to the correct set. 

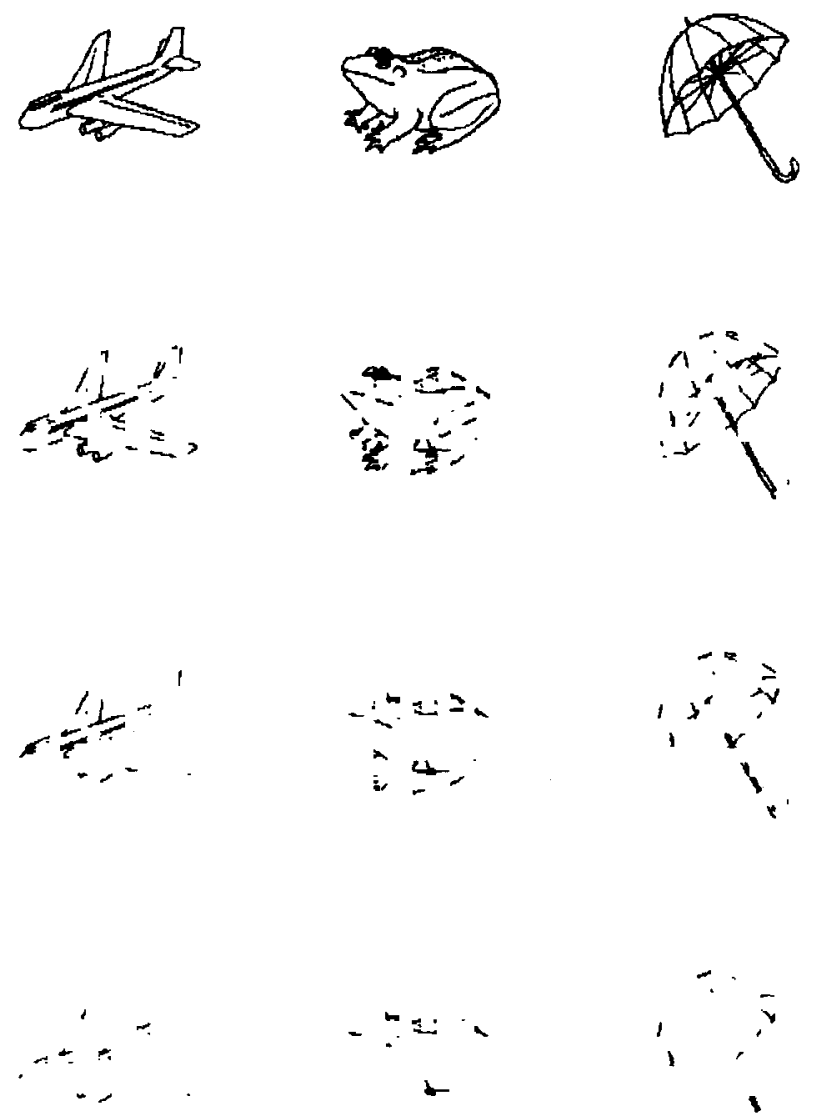

Figure 1. Examples of fragmented images at levels 8 (complete), 6,4 , and 2 .

The program disk contains the program for running the memory test, a customized library of Clear Lake Research statements that access machine language routines, the program for updating variant files, and a program for accessing the stored data from individual subjects. The analysis program lets the user print out data from individual subjects and write the data to the clipboard, from which it can be pasted to a spreadsheet. The data consists of thresholds for each picture within the training, new, and old sets, sorted by identification number (and thus alphabetically by the name of the picture). The mean thresholds for the training, old, and new conditions are also computed. Incorrect responses are not stored.

Figure 1 shows three examples of fragmented pictures at selected levels of fragmentation. The levels shown, from top to bottom, are 8 (complete picture), 6,4 , and 2 (one level above the most fragmented level).

\section{The Memory Test}

We created five forms of the memory test by dividing the set of 150 pictures into 10 sets of 15 pictures equated on name agreement, rated familiarity, and rated complexity according to the Snodgrass and Vanderwart norms, and with approximately the same distribution of exemplars across categories. A particular form of the test uses two sets of 15 pictures, one set for the training or old stimuli, and one set for the new stimuli. The five forms are numbered 1 through 5 , and the two sets within each form are labeled $a$ and $b$. By using $a$ or $b$ as training stimuli (and b or a as new stimuli), a total of 10 forms can be created, although a particular subject can be exposed to only five forms (e.g., 1a or 1b) without repeating items across tests.

In the first phase of the memory test, a form and set are selected by the experimenter to serve as old stimuli and these pictures are randomly ordered by the program. Picture identification thresholds are measured by the ascending methods of limits. The most fragmented version of each picture is presented and the subject either attempts to identify it (by typing its name on the keyboard) or presses the return key to go on to the next level. A name is correct if it matches one of the listed names for that concept stored in the variant files. Incorrect names are not penalized, so there is no penalty for guessing. The experimenter can update a variant file by adding an additional name that he or she wishes to be considered as correct. The variant files were constructed by using the names that occurred more than once in the Snodgrass and Vanderwart norms, and then adding those that more than one subject responded with in the normative study reported here.

When the picture has been correctly identified, the program stores the level of fragmentation at which the training item was identified and moves on to the next picture in the random sequence. After all 15 pictures have been correctly identified, subjects can be given a distractor task (we used a 10-min cancellation-of-9s paper-and-pencil test), or subjects can proceed immediately to the test phase.

In the test phase, subjects are presented with a test set of 30 pictures, presented in a random order determined by the program. The test set consists of the 15 pictures used in the training series (the old set) and a set of 15 new pictures (the new set). Subjects go through the same procedure of identifying each picture by typing in its name or pressing the return key to go to the next level. As in the training phase, there is no penalty for guessing.

The data of interest are the identification thresholds for the training stimuli during the first phase, and the identification thresholds for the old and new stimuli during the test phase. Learning the task (skill learning) is indexed by a decrease in thresholds between the training and new stimuli (training minus new). Learning the items (perceptual learning) is indexed by a decrease in thresholds between the new and old items in the test (new minus old). The perceptual learning measure thus excludes improvement in performance due to skill learning.

The memory testing program provides the option of either presenting or not presenting the complete picture in the training phase. This option could be implemented to compare the effects of complete stimulus priming on subsequent test identification thresholds. In the normative study reported here, we did not present the complete picture during training (the complete picture is never presented during testing). 
Table 1

Mean Threshold Values by Set and Training Condition

\begin{tabular}{cccccc}
\hline Set & Train & New & Old & Train-New* & New-Old $t$ \\
\hline 1a & 5.23 & 4.49 & 3.25 & 0.75 & 1.23 \\
lb & 4.77 & 5.19 & 2.66 & -0.42 & 2.53 \\
2a & 5.02 & 5.27 & 2.75 & -0.25 & 2.51 \\
2b & 4.87 & 4.38 & 2.28 & 0.49 & 2.10 \\
3a & 4.52 & 4.33 & 2.33 & 0.19 & 2.00 \\
3b & 4.57 & 4.38 & 2.46 & 0.19 & 1.92 \\
4a & 4.88 & 4.41 & 2.61 & 0.47 & 1.80 \\
4b & 4.92 & 4.78 & 2.92 & 0.14 & 1.86 \\
5a & 4.39 & 5.11 & 2.56 & -0.72 & 2.55 \\
5b & 5.07 & 3.89 & 2.81 & 1.19 & 1.08 \\
Mean & 4.82 & 4.62 & 2.66 & 0.20 & 1.96 \\
\hline
\end{tabular}

*Measure of skill learning. $†$ Measure of perceptual learning.

\section{RESULTS OF THE NORMATIVE STUDY}

In order to test the procedure and to obtain normative data on the 150 pictures, we ran the basic memory task with 100 volunteer subjects who participated to fulfill a course requirement in introductory psychology. Subjects were randomly assigned to each of the 10 versions of the test ( 5 forms $\times 2$ sets) until 10 subjects had participated in each version.

Table 1 presents mean thresholds for the train, new, and old stimuli for the $a$ and $b$ versions of each of the five forms. The task is scored so that good performance is accompanied by low numbers. A threshold of 1 means the stimulus was identified at its most fragmented level, and a threshold of 8 means the stimulus was identified at its most complete level. Sets a and b within each form are paired so that one set serves alternately as the training and the new set of stimuli. So, for example, $1 \mathrm{~b}$ serves as the new stimuli set for $1 \mathrm{a}$ and vice versa.

It is clear that subjects improve with practice on the task (there is an average decrease of 0.20 level from train to new across sets). However, not all subsets show this decrease. Sets $1 b, 2 a$, and $5 a$ all show an increase in threshold between the train and new sets. In two of the three cases, the problem has to do with initial inequality between sets. Set 1a is harder than $1 b$, and Set $5 b$ is harder than 5a, so subjects' skill learning is masked by set differences. It is also clear that subjects improve markedly on the repeated items, with an average decrease in threshold from new to old of almost two fragmentation levels. All forms and sets within forms show this decrease.

Several analyses of variance were performed in order to separately investigate the learning component and the form-specific effects. To evaluate the learning component with minimal influence of set differences, we combined the $a$ and $b$ forms of each test (to eliminate differences between Set a and Set $b$ ) and performed a 3 (training) $\times 5$ (form) mixed analysis of variance. There was a significant effect of form $[F(4,95)=3.55, p<.01]$, a highly significant effect of training $[F(2,190)=142.65$, $p<.001]$, and no interaction between form and training $[F(8,190)=1.31]$. The results of Tukey HSD post hoc comparisons showed that both memory components were significant at the .01 level. Subjects showed significant skill learning, because new thresholds were significantly lower than train thresholds. And subjects showed significant perceptual learning, because old thresholds were significantly lower than new thresholds. The only significant form difference was that between Form 1 and Form 3. The lack of a significant interaction is not particularly surprising, given that across forms, item effects are counterbalanced between the train and new sets, and thus average out when combined. Figure 2 presents the average thresholds as a function of training for each form.

To investigate differences between $a$ and $b$ sets within forms in more detail, we carried out individual 3 (training) $\times 2$ (set: a vs. b) analyses of variance on each of the five forms. An ideal form would show no significant effect of set, a significant effect of training, and no interaction of set with training. All forms showed a significant effect of training [all $\left.F_{\mathrm{s}}(2,36)>215\right]$. Only two forms (3 and 4) showed no effect of set and no interaction. Forms 1 and 5 both showed an insignificant effect of set but a highly $(p<.0001)$ significant interaction, while Form 2 showed both a significant effect of set $(p=.006)$ and a significant interaction $(p=.0013)$.

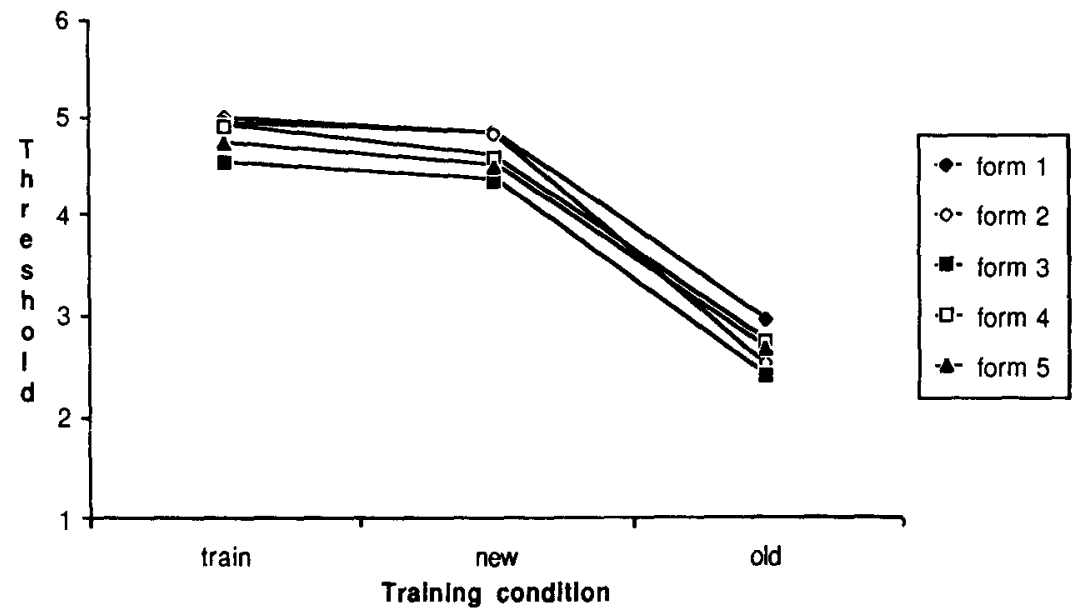

Figure 2. Mean identification thresholds for the three training conditions by form $(1=$ most fragmented and $8=$ complete). 
In order to optimize the use of this memory test, particularly for use in clinical trials (in which several equivalent forms of a test are required to evaluate treatment effects), it will be necessary to adjust items within Forms 1,2 , and 5 so that they are more nearly equated for difficulty of initial identification in their a and b sets. We are presently engaged in doing just that. In the meantime, however, Forms 3 and 4 may be used with a fair amount of confidence that items within their $a$ and $b$ sets are equivalent. This provides for two independent forms for within-subjects designs and four quasi-independent forms for between-subjects designs (i.e., $3 a, 3 b, 4 a$, and $4 b$ ).

\section{DISCUSSION}

The experimental task presented here makes it possible to separately measure two components of memory: skill learning, measured by an improvement on the task across different items, and perceptual learning, measured by an improvement on the task across the same items. Skill learning has also been called procedural memory in the literature, and perceptual learning has also been called implicit memory or perceptual fluency. Although the skill learning component is significant in this task, perceptual learning is almost an order of magnitude larger. However, we do not believe that the perceptual learning component as measured in this task is independent of episodic memory influence. Some of the perceptual learning effect could be due to explicit episodic memory for the fragments themselves (because the same fragments are presented on the old trials as were presented on the training trials), or to memory for the names of the training items that occur as lucky guesses during test. We are presently investigating both of these possibilities.

\section{AVAILABILITY OF THE MEMORY TEST}

A disk (800K) containing 250 of the 260 Snodgrass and Vanderwart pictures as MBASIC files is available from the first author for $\$ 5$. The complete perceptual memory test is available on six $800 \mathrm{~K}$ disks. One disk contains the programs for running the experiment and analyzing the data, and the remaining five disks contain the five memory test forms. These are available from the first author for $\$ 30$. The user must have MBASIC (version 2.1 or higher) to run these programs. In addition, Brooks (1985) provides all 260 Snodgrass and Vanderwart pictures on two disks as MacPaint files.

\section{REFERENCES}

Brooks, J. O., III. (1985). Pictorial stimuli for the Apple Macintosh computer. Behavior Research Methods, Instruments, \& Computers, 17, 409-410.

Corkin, S. (1982). Some relationships between global amnesias and the memory impairments in Alzheimer's disease. In S. Corkin, K. L. Davis, J. H. Growdon, E. Usdin, \& R. J. Wurtman (Eds.), Alzheimer's disease: A report of progress (pp. 149-164). New York: Raven Press.

GoLLIN, E. S. (1960). Developmental studies of visual recognition of incomplete objects. Perceptual \& Motor Skills, 11, 289-298.

Graf, P., Squire, L. R., \& Mandler, G. (1984). The information that amnesic patients do not forget. Journal of Experimental Psychology: Learning, Memory, \& Cognition, 10, 164-178.

JACOBY, L. L. (1983). Perceptual enhancement: Persistent effects of an experience. Journal of Experimental Psychology: Learning, Memory, \& Cognition, 9, 21-38.

JACOBY, L. L., \& DALLAS, M. (1981). On the relationship between autobiographical memory and perceptual learning. Journal of Experimental Psychology: General, 3, 306-340.

JACOBY, L. L., \& WITHERSPOON, D. (1982). Remembering without awareness. Canadian Journal of Psychology, 36, 300-324.

SNODGRASS, J. G., \& VANDERWART, M. (1980). A standardized set of 260 pictures: Norms for naming agreement, familiarity, and visual complexity. Journal of Experimental Psychology: Human Learning \& Memory, 6, 174-215.

Tulving, E. (1985). How many memory systems are there? American Psychologist, 4, 385-398.

WaRrington, E. K., \& WeISKRantz, L. (1968). New method of testing long-term retention with special reference to amnesic patients. Nature, 217, 972-974.

Vokey, J. R., BaKer, J. G., Hayman, G., \& Jacoby, L. L. (1986). Perceptual identification of visually degraded stimuli. Behavior Research Methods, Instruments, \& Computers, 18, 1-9. 